\title{
Variabilidade e Previsão Climática de Vazões na Margem Esquerda da Bacia do Alto Paraná (Brasil)
}

\author{
Ilya S. Kim \\ Instituto Tecnológico SIMEPAR - iskim@simepar.br \\ Nelson L. Dias \\ Lemma - Laboratório de Estudos em Modelagem e Monitoramento Ambiental - UFPR/LAPAR/SIMEPAR \\ nldias@simepar.br
}

Recebido: 02/02 - revisão: 07/02 - aceito: 02/03

\section{RESUMO}

Por meio de análise fatorial foi feita uma regionalização da margem esquerda da bacia do Alto Paraná (Brasil) em duas sub-bacias hidrologicamente homogêneas do ponto de vista dos processos de formação de vazões. Também, através de análise fatorial, foi feito um agrupamento dos meses em estações do ano nas sub-bacias. Foram identificadas as estações do ano que são mais importantes para a composição da vazão anual. Foi feita uma analise da influência dos fenômenos El Niño e La Niña para as formações de vazõos na margem esquerda da bacia do Alto Paraná (Brasil). Além disso, por meio dos métodos de analogia e correlação canônica e com uso de informações sobre a temperatura da superfície do mar e a temperatura do ar em pontos selecionados do Hemisfério Sul (com correlaçöes insignificantes entre si) foram desenvolvidos e validados modelos de previsão climática mensal de vazões.

Palavras-chave: regionalização; previsão climática.

\section{INTRODUÇÃO}

O conhecimento da variabilidade e da variação climática das vazões é útil para planejamento da operação de sistema de recursos hídricos, setor de produção de energia, defesa civil e etc.). Porém, a variabilidade climática das vazões superficiais medidas nas calhas dos rios depende das forçantes atmosféricas climáticas regionais. Estas por sua vez estão relacionadas com a variação climática global ou, pelo menos, hemisférica. Mechoso e Iribarren (1992) estudaram as relações entre a Oscilação Sul e as vazões no sudeste da América do Sul, enquanto que Guietto e Berry (1996) apresentam relações entre as vazões anuais, trimestrais e mensais nos rios do sudeste da América do Sul e índices do El Niño. Recentemente, em Gente et al. (1998) foram analisadas as tendências de crescimento nas vazões dos rios do sudeste da América do Sul; Tucci e Damiani (1994) estudaram o efeito de mudanças climáticas sobre o regime de vazões do rio Uruguai.

No presente trabalho a variabilidade e variação climática das vazões anuais e mensais foram estudadas buscandose das regiões hidrologicamente homogêneas (do ponto de vista dos processos de formação de vazões). A regionalização da margem esquerda da bacia do rio Paraná em duas subbacias homogêneas foi feita por análise fatorial com o uso dos dados de vazão anual para o período de 1931-2000. Subseqüentemente, a análise fatorial é usada em cada região hidrologicamente homogênea para agrupar meses homogêneos, o que é equivalente à identificação de padrões sazonais, assim como para identificar as estações do ano (grupos de meses) que são mais importantes na composição da vazão média anual. Em seguida, faz-se uma análise das relações entre os fenômenos El Niño e La Niña com as vazões na margem esquerda do Paraná. Além disso, por meio dos métodos de analogia e correlação canônica, e com o uso de dados de temperatura da superfície do mar e temperatura do ar em pontos selecionados do Hemisfério Sul, foram desenvolvidos e validados modelos de previsão climática mensal para as vazões.

\section{DADOS E METODOLOGIA}

\section{Dados}

Neste estudo foram usados os dados de estações hidrológicas situadas em regiões mais a montante possível na margem esquerda do rio Paraná, onde as vazões são menos afetadas pela regularização devido à construção de barragens. Os dados de vazão mensal das estações hidrológicas de Corumbá (rio Corumbá), Emborcação (rio Paranaíba), Nova Ponte (rio Araguari), Porto Colômbia (rio Grande), Barra Bonita (rio Tietê), Capivara (rio Paranapanema) e Salto Osório (rio Iguaçu) do período 1931-2000 da bacia do rio 
Paraná foram fornecidos pelo Operador Nacional do Sistema Elétrico (Figura 1).

Também foram utilizados os dados de temperatura da superfície do mar (TSM) e temperatura do ar $\left(\mathrm{T}_{\mathrm{ar}}\right)$ em 30 pontos (15 de TSM e 15 de $\mathrm{T}_{\mathrm{ar}}$ ) selecionados do Hemisfério Sul para o período 1940-2000 (Kim, 1998) Figura 2. Estes pontos não apresentam correlações espaciais significativas entre si, sendo que cada um deles é representativo das temperaturas junto à superfície de uma certa região no Hemisfério Sul. Considerados em conjunto, esses trinta pontos caracterizam grosso modo os campos de TSM e de $\mathrm{T}_{\mathrm{ar}}$ nos continentes do Hemisfério Sul. Os dois pontos no Oceano Pacíficos estão próximos as regiões equivalentes aos índices ElNiño 3 e ElNiño 4. O ElNiño 3 apresenta TSM dentro do quadrado $5^{\circ} \mathrm{N}-5^{\circ} \mathrm{S}$ e $150^{\circ} \mathrm{W}-90^{\circ} \mathrm{W}$ e El Niño 4 a TSM dentro do quadrado $5^{\circ} \mathrm{N}-5^{\circ} \mathrm{S}$ e $160^{\circ} \mathrm{E}-150^{\circ} \mathrm{W}$. Esta é uma observação importante porque o uso destes pontos em modelos de previsão climática de vazão permite incorporar automaticamente os efeitos El Niño e La Niña.

Os dados de TSM e $\mathrm{T}_{\mathrm{ar}}$ são sempre usados em conjunto indistintamente, ou seja: os modelos que foram aplicados não distinguem os dois tipos de variáveis.

\section{Métodos}

Foi aplicada a análise fatorial, que permite substituir os dados iniciais por um número menor de variáveis (fatores), que são a combinação linear dos dados iniciais. Matematicamente, a análise fatorial pressupõe uma relação da forma:

$$
Y_{i, k}=\sum_{p=1}^{N_{F}} A_{i, p} F_{p, k}+\varepsilon_{i, k}
$$

onde $\mathrm{Y}_{\mathrm{i}, \mathrm{k}}$ é a vazão anual no posto i e ano $\mathrm{k}, \mathrm{F}_{\mathrm{p}, \mathrm{k}}$ é o p-ésimo fator comum - ou simplesmente, fator - para o ano k, $\mathrm{A}_{\mathrm{i}, \mathrm{p}}$ é a carga fatorial do posto i para o fator $\mathrm{p}$, sendo proporcional ao coeficiente de correlação entre $\mathrm{Y}_{\mathrm{i}, \mathrm{k}}$ e $\mathrm{F}_{\mathrm{p}, \mathrm{k}}$, e o número de fatores $\mathrm{N}_{\mathrm{F}}$ deve ser menor que o número de postos fluviométricos $\mathrm{N}_{\mathrm{Q}}: \mathrm{N}_{\mathrm{F}}<\mathrm{N}_{\mathrm{Q}}$. O modelo admite um erro $\varepsilon_{\mathrm{i}, \mathrm{k}}$ que não esta correlacionado com fatores $\mathrm{F}_{\mathrm{p}, \mathrm{k}}$. Os métodos normalmente utilizados para a obtenção de $\mathrm{N}_{\mathrm{F}}$ assim como $\mathrm{A}_{\mathrm{i}, \mathrm{p}}$ 's e $\mathrm{F}_{\mathrm{p}, \mathrm{k}}$ 's são relativamente sofisticados, e o problema não possui solução única nestas duas últimas variáveis (Kendall, 1980). Neste trabalho, foi utilizado o Método do Fator Principal (Gnanadesikan, 1997) para a realização da análise fatorial. Neste método, é importante definir a matriz de covariância de Y:

$$
\Sigma=\frac{1}{n-1}[Y-\bar{Y}] \cdot[Y-\bar{Y}]^{\prime}
$$

onde n é o número de colunas de $\mathrm{Y}$ (no caso de vazões anuais, n é o número de anos do histórico de vazões) e (')

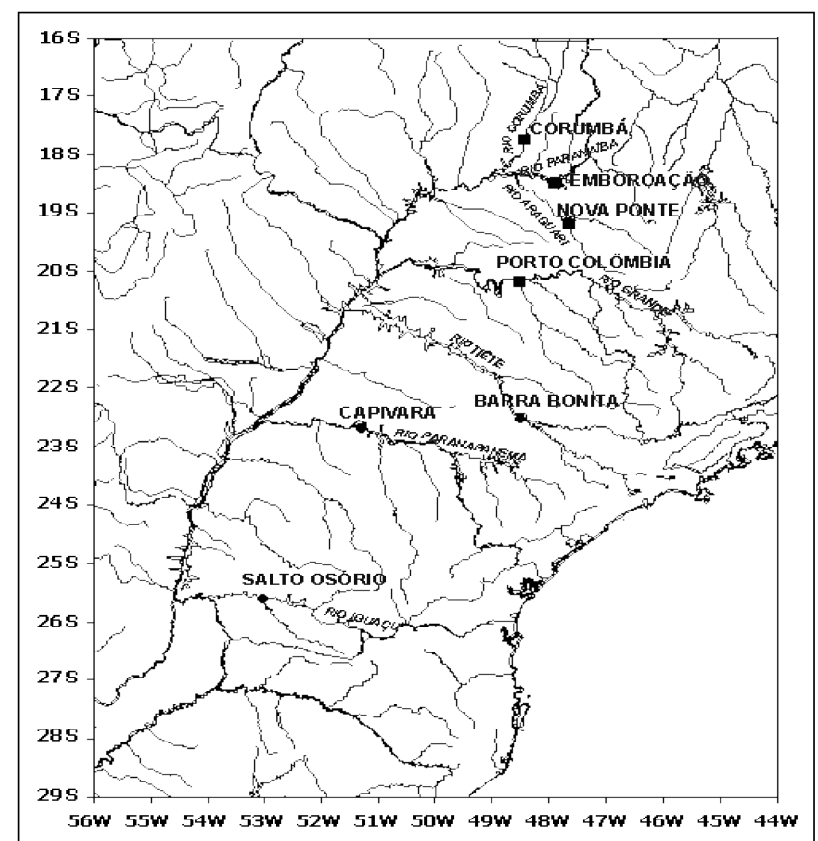

Figura1. Mapa da bacia do rio Paraná com disposições das estações hidrológicas.

indica a matriz-transposta, a matriz-diagonal auxiliar D tem seus elementos dados por:

$$
\mathrm{D}_{\mathrm{i}, \mathrm{j}}=\delta_{\mathrm{i}, \mathrm{j}} \frac{1}{\sqrt{\Sigma_{\mathrm{i}, \mathrm{i}}}}
$$

e a matriz de correlação:

$$
\mathrm{R}=\mathrm{D} \cdot \Sigma \cdot \mathrm{D}
$$

uma vez que $\mathrm{N}_{\mathrm{F}}$ e a matriz $\mathrm{A}$ tenham sido estimados pelo método do Fator Principal, F pode ser estimada via (Gnanadesikan, 1997):

$$
\hat{\mathrm{F}}=\mathrm{A}^{\prime} \cdot \mathrm{R}^{-1} \cdot \mathrm{D} \cdot[\mathrm{Y}-\overline{\mathrm{Y}}]
$$

As relações entre os fenômenos ElNiño e La Niña e as vazões dos rios na margem esquerda da bacia do rio Paraná foram investigadas de acordo com uma proposta de classificação de datas e intensidades de El Niño/La Niña (Kim, 2001). Neste trabalho, foi desenvolvido um método de definição tanto das datas quanto das intensidades desses fenômenos com o uso de séries dos índices El Niño 1-2, ElNiño 3, El Niño 3.4 e El Niño 4, por meio do uso de sete intervalos determinados através dos valores dos quantis de $2,5 \%$, $16 \%, 33 \%, 67 \%, 84 \%$ e $97,5 \%$.

1. < quantil $2,5 \%$;

2. $>$ quantil $2,5 \%$ e $<$ quantil $16 \%$; 


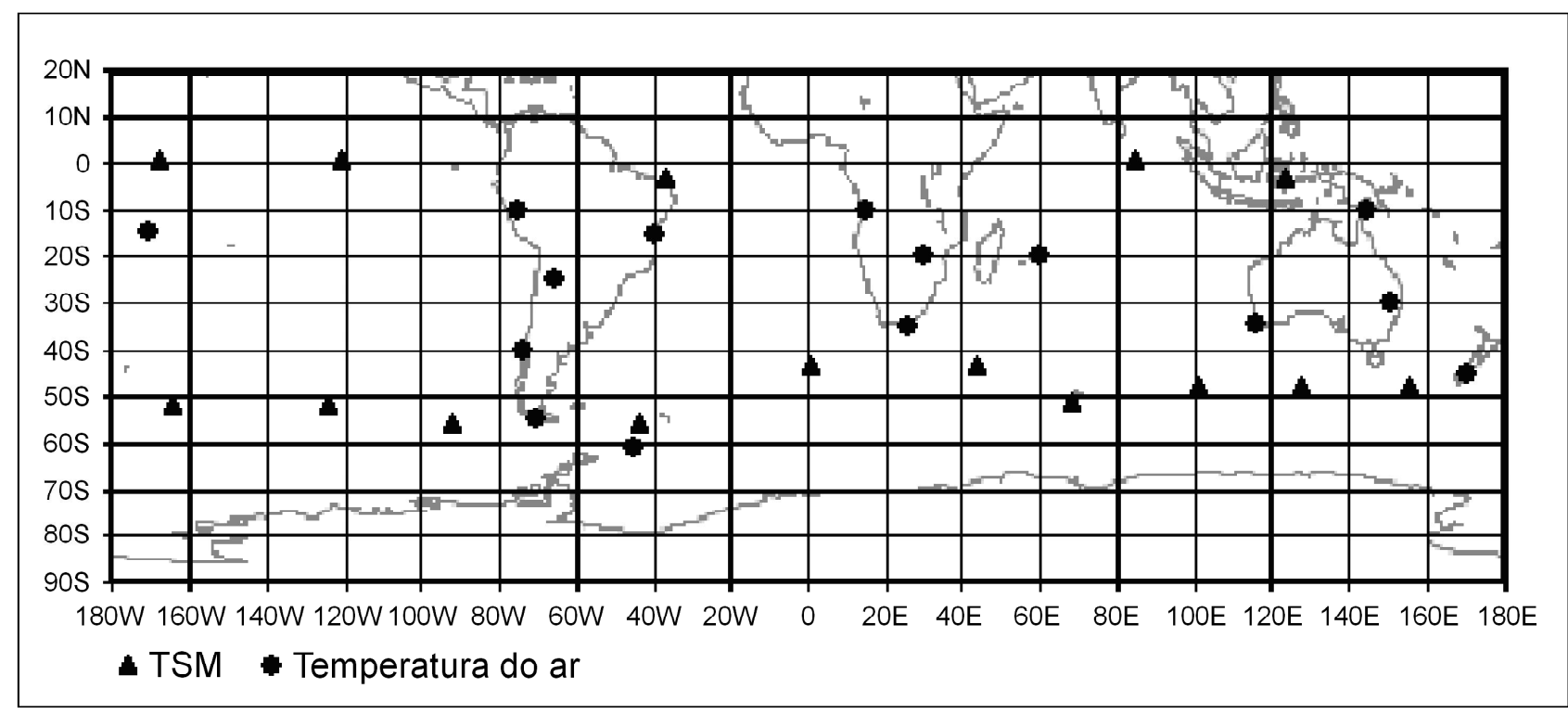

Figura 2. Localização dos pontos de TSM e temperatura do ar no Hemisfério Sul.
3. > quantil $16 \%$ e < quantil $33 \%$;
4. > quantil $33 \%$ e $<$ quantil $67 \%$;
5. $>$ quantil $67 \%$ e $<$ quantil $84 \%$;
6. $>$ quantil $84 \%$ e $<$ quantil $97,5 \%$;
7. $>$ quantil $97,5 \%$.

Os intervalos extremos (1 e 7) correspondem aproximadamente a parte da distribuição de freqüência fora do valor do dois desvios-padrão. Os intervalos 2 e 6 correspondem aproximadamente do valor entre um e dois desvios-padrão. O intervalo 4 corresponde à norma.

Com a utilização destes intervalos foi feita a identificação dos valores de índices El Niño, ou seja se durante 5 meses ou mais foi encontrado:

i. O intervalo 7 - foi fixado o fenômeno $\mathrm{El}$ Niño forte

ii. O intervalo 6 (alguns meses podem ter o intervalo 7) foi notado El Niño moderado

iii. O intervalo 5 (alguns meses podem ter o intervalo 6 ou 7) - foi marcado El Niño fraco.

iv. O intervalo 3 (alguns meses podem ter o intervalo 2 ou 1) - foi notada La Niña fraca.

v. O intervalo 2 (alguns meses podem ficar com intervalo 1) - foi fixada La Niña moderada.

vi. O intervalo 1 - foi marcada La Niña forte.

Portanto, se durante 5 meses consecutivos ou mais encontramos os intervalos 5, 6 e 7 temos o fenômeno El Niño, se forem os intervalos 1, 2 e 3, esta correspondendo do fenômeno La Niña. Este modo permitiu obter não só datas de El Niño e La Niña mas também suas intensidades (fraca, moderada e forte) de forma objetiva. As datas obtidas possuem boa correspondência com aquelas encontradas por outros autores (Trenberth, 1997, por exemplo).

\section{Tratamento de dados}

Seja $Q_{\mathrm{i}, \mathrm{j}, \mathrm{k}}$ a vazão no posto fluviométrico i, no mês $1 \mathrm{e}$ no ano k. A vazão média do posto i no mês j para o período do histórico hidrológico de $\mathrm{N}_{\mathrm{H}}$ anos é:

$$
\overline{\mathrm{Q}}_{\mathrm{i}, \mathrm{j}}=\frac{1}{\mathrm{~N}_{\mathrm{H}}} \sum_{\mathrm{k}=1}^{\mathrm{N}_{\mathrm{H}}} \mathrm{Q}_{\mathrm{i}, \mathrm{j}, \mathrm{k}}
$$

Os dados de vazão podem ser normalizados calculando-se as anomalias mensais em \%:

$$
\eta_{i, j, k}=\frac{Q_{i, j, k}}{\bar{Q}_{i, j}} \times 100
$$

As médias das anomalias mensais de vazão no mês $\mathrm{j} e$ anos k para toda a região são calculadas através da equação:

$$
\bar{\eta}_{\mathrm{j}, \mathrm{k}}=\frac{1}{\mathrm{~N}_{\mathrm{Q}}} \sum_{\mathrm{j}=1}^{\mathrm{N}_{\mathrm{Q}}} \eta_{\mathrm{i}, \mathrm{j}, \mathrm{k}},
$$

onde $\mathrm{N}_{\mathrm{Q}}$ é o número de postos fluviométricos utilizados.

As TSM's e os valores de $\mathrm{T}_{\text {ar }}$ nos trinta pontos escolhidos do Hemisfério Sul serão doravante denominados "temperaturas" e denotados por $\mathrm{T}_{\mathrm{i}, \mathrm{j}, \mathrm{k}}$ para a estação $\mathrm{i}$ (que pode ser um ponto no continente, quando então se trata de $\mathrm{T}_{\mathrm{ar}}$, ou no mar, quando se trata de TSM), mês j e ano k, de forma semelhante às vazões. A sua média para o ponto i no mês $j$ é:

$$
\overline{\mathrm{T}}_{\mathrm{i}, \mathrm{j}}=\frac{1}{\mathrm{~N}_{\mathrm{M}}} \sum_{\mathrm{j}=1}^{\mathrm{N}_{\mathrm{M}}} \mathrm{T}_{\mathrm{i}, \mathrm{j}, \mathrm{k}}
$$

onde $\mathrm{N}_{\mathrm{M}}$ é o número de anos do histórico meteorológico; o seu desvio-padrão no ponto i e mês j é, analogamente, dado 
por:

$$
\mathrm{s}_{\mathrm{T}_{\mathrm{i}, \mathrm{j}}}=\sqrt{\frac{1}{\mathrm{~N}_{\mathrm{M}}-1} \sum_{\mathrm{k}=1}^{\mathrm{N}_{\mathrm{M}}}\left(\mathrm{T}_{\mathrm{i}, \mathrm{j}, \mathrm{k}}-\overline{\mathrm{T}}_{\mathrm{i}, \mathrm{j}}\right)^{2}}
$$

Os valores de $\mathrm{T}_{\mathrm{ar}}$ e TSM podem ser consideravelmente diferentes, e por isso foram normalizados por suas médias e desvios-padrão, produzindo-se uma série de anomalias de temperatura com média zero e desvio-padrão igual a um, dadas por:

$$
x_{i, j, k}=\frac{T_{i, j, k}-\bar{T}_{i, j}}{s_{T_{i, j}}}
$$

Note-se que as definições de "anomalia de vazão" e "anomalia de temperatura" utilizadas neste trabalho são diferentes. Um valor de 100\% significa uma vazão mensal igual à média de longo período, enquanto que um valor igual a zero tem o mesmo significado para a temperatura.

Para descrever os algoritmos de previsão da vazão mensal para 1 e 2 meses, é mais conveniente trabalhar com o índice corrido do mês no histórico:

$$
\mathrm{t}=(\mathrm{k}-1) \mathrm{x} 12+\mathrm{j}
$$

do que com o ano k e o mês j. Onde for pertinente, será subentendido daqui para frente que $\eta_{i, t}=\eta_{i, j, k}$ e $x_{i, t}=x_{i, j, k}$, com (12) valendo entre os índices t, j e k. Dois modelos para a previsão climática mensal das anomalias de vazão $\eta$ são descritos a seguir.

1. Com o uso do método de analogias. Neste método, o estado presente da atmosfera é caracterizado de forma simplificada pelo conjunto de $\mathrm{N}_{\mathrm{M}}=30$ valores de anomalias de temperatura normalizada na forma dos $\mathrm{x}_{\mathrm{i}, \mathrm{j}, \mathrm{k}}$. Mais especificamente, seja:

$$
\mathrm{X}^{\mathrm{obs}}=\left\{\mathrm{x}_{\mathrm{i}, \mathrm{j}_{\mathrm{p}}, \mathrm{k}_{\mathrm{p}}}\right\}, \mathrm{i}=1, \ldots, \mathrm{N}_{\mathrm{M}}
$$

onde o índice P dá o estado "presente" de anomalias de temperatura não Hemisfério Sul, para o mês e ano correntes $j_{\mathrm{p}}$ e $k_{\mathrm{p}}$. Serão calculadas para todos os meses do histórico contíguo ao mês corrente $\left(j \in\left\{j_{P}, j_{P}-1, j_{P}+1\right\}\right)$, em todos os anos do histórico $\left(\mathrm{k}<\mathrm{k}_{\text {atual }}\right)$ as distâncias euclidianas da situação atual:

$$
S_{j, k}=\sqrt{\frac{1}{N_{M}} \sum_{i=1}^{N_{M}}\left(x_{i, j, k}-x_{i, j_{p}, k_{p}}^{o b s}\right)^{2}}
$$

Em seguida, ordena-se os valores $S_{j, k}$ e obtém-se os $\mathrm{L}=20$ menores valores correspondentes às situações históricas mais "parecidas" com o estado presente da atmosfera e do oceano.
Após, serão calculadas as diferenças das anomalias de vazões mensais entre datas das análogas t e 1 e 2 meses à frente $\mathrm{t}+1 \mathrm{e} \mathrm{t}+2$ :

$$
\begin{aligned}
& \delta \eta_{i, 1}^{+1}=\eta_{i, t+1}-\eta_{i, t} \\
& \delta \eta_{i, 1}^{+2}=\eta_{i, t+2}-\eta_{i, t}
\end{aligned}
$$

As previsões de anomalia para 1 e 2 meses à frente do mês atual são obtidas via:

$$
\begin{aligned}
& \hat{\eta}_{\mathrm{i}, \mathrm{t}_{\mathrm{p}}+1}=\eta_{\mathrm{i}, \mathrm{t}_{\mathrm{p}}}+\frac{1}{\mathrm{Q}} \sum \delta \eta_{\mathrm{i}, 1}^{+1} \\
& \hat{\eta}_{\mathrm{i}, \mathrm{t}_{\mathrm{p}}+1}=\eta_{\mathrm{i}, \mathrm{t}_{\mathrm{p}}}+\frac{1}{\mathrm{Q}} \sum \delta \eta_{\mathrm{i}, 1}^{+2}
\end{aligned}
$$

Em outras palavras, a previsão para $\eta$ é simplesmente o estado atual mais a média das tendências observadas no histórico sobre as L situações meteorologicamente mais parecidas (num sentido muito aproximado, bem entendido) com o estado atual da atmosfera.

2. Com o uso das correlações canônicas. Neste método, dados dois vetores x e y, encontra-se os dois conjuntos de combinações lineares dos elementos de x e y que apresentam a máxima correlação; em seguida, entre os conjuntos de combinações lineares respectivamente ortogonais a estes dois, repete-se o processo, e assim sucessivamente. Em geral, se p e q são as dimensões de x e y, e se $\mathrm{p}<\mathrm{q}$, é possível extrair p pares de combinações lineares desta forma. As combinações lineares assim obtidas são denominadas "variáveis canônicas", e suas correlações são denominadas "correlações canônicas" (Kendall, 1980; Gnanadesikan, 1997; Barnet e Pzeisendorfer, 1987).

Os coeficientes de correlação canônicos entre preditantes x e preditores $\eta$ com diferentes defasagens no tempo foram usados para desenvolver o modelo de previsão climática (Barnet e Pzeisendorfer, 1987).

A avaliação das previsões de anomalias das vazões mensais obtidas através dos modelos descritos acima foi feita por meio do cálculo do índice de acerto:

$$
D_{i, t}=\frac{\left|\hat{\eta}_{i, t}-\eta_{i, t}\right|}{s_{\eta_{i}}}
$$

onde $\hat{\eta}_{i, t}$ e $\eta_{i, t}$ são as anomalias de vazão prevista e observada, respectivamente, e $s_{\eta_{i}}$ é o desvio-padrão da anomalias mensais de vazão calculado sobre o histórico de meses corridos. Se $\mathrm{D}_{\mathrm{i}, \mathrm{t}}<1$ (previsão a menos de um desvio-padrão da observação), considera-se que a previsão foi acertada, e se $D_{i, t}>1$, considera-se que a previsão foi errada. 


\section{REGIONALIZAÇÃO}

A área da margem esquerda da bacia do rio Paraná está sob a influência de características climáticas bastante diferentes, o que leva a diferentes condições de formações das vazões. Uma forma de se avaliar a variabilidade espacial das vazões é procurar as sub-bacias em termos de regiões hidrologicamente homogêneas ou seja, regionalizar a bacia estudada. Foram calculadas as vazões anuais para o ano hidrológico, que se inicia em outubro e termina em setembro para o período 1931-2000. Para efetuar a regionalização, foi utilizada a análise fatorial descrita na seção 2.

A análise fatorial dividiu a região estudada em duas regiões: a primeira localizada acima de $22^{\circ} \mathrm{S}$ (zona tropical) foi formada pelo grupo das estações hidrológicas Corumbá, Emborcação, Nova Ponte e Porto Colômbia e a segunda região fica abaixo de $22^{\circ} \mathrm{S}$ (zona subtropical), tendo sido formada pelas estações Barra Bonita, Capivara e Salto Osório (Figura 1). Na Tabela 1 estão mostrados os valores das cargas fatoriais (ou coeficientes de correlação entre as vazões anuais nas estações e fatores obtidos). Observa-se na Tabela 1 , os valores das cargas fatoriais das estações tanto para a primeira região quanto para a segunda região ficaram acima de 0,7 , o que significa que a separação das regiões hidrologicamente homogêneas é bastante nítida. Os resultados da análise fatorial possuem uma boa correspondência com os processos atmosféricos.

Na Figura 3 são mostradas as séries temporais dos fatores que caracterizam as duas regiões para o período 1931-2000, obtidas através da Equação (5). Como podemos ver na Figura 3, o fator que caracteriza a região não teve tendência linear até o ano de 1980, sendo que nos últimos 15 a 20 anos as vazões por ele caracterizadas têm diminuído. Uma tendência oposta se observa na região 2 , onde o fator 2 (fica normalmente abaixo da média global até o fim dos anos 1960 e início dos anos 1970, depois fica acima da média total).

A Figura 4 mostra a distribuição das vazões mensais durante o ano na estação de Nova Ponte, que caracteriza a primeira região, e na estação de Capivara, que caracteriza a segunda região. A vazão em Nova Ponte tem o valor máximo em fevereiro e o mínimo em setembro. $\mathrm{Na}$ estação Capivara, a vazão tem três máximos, sendo o principal em fevereiro e os secundários em outubro e junho, e três mínimos, em abril, agosto e novembro.

Foram calculadas as anomalias mensais em porcentagem das normais climáticas do período 1931-2000 nas estações estudadas, conforme de Equação (7). Em seguida foram calculadas as médias de anomalias mensais para cada região com o uso da Equação (8), e foi obtida uma série de anomalias mensais para cada uma das regiões 1 e 2 . Utilizou-se, então, analise fatorial para identificar os meses estatisticamente semelhantes (no sentido de sua variabilidade temporal) nestas duas séries regionais. Nas estações da pri-
Tabela 1. Valores das cargas fatoriais ou coeficientes de correlação entre as vazões anuais e fatores obtidos.

\begin{tabular}{lcc}
\hline Estação & Fator 1 & Fator 2 \\
\hline Corumbá & 0,889 & 0,000 \\
Emborcação & 0,952 & 0,095 \\
Porto Colômbia & 0,713 & 0,459 \\
Nova Ponte & 0,901 & 0,224 \\
Salto Osório & $-0,066$ & 0,883 \\
Barra Bonita & 0,275 & 0,822 \\
Capivara & 0,072 & 0,948 \\
\hline
\end{tabular}

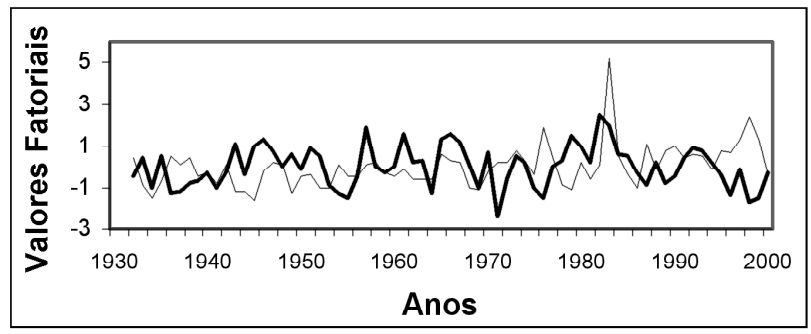

Figura 3. Variações dos fatores 1 e 2 que descrevem as variações dos recursos hídricos na região 1 (negrito) $\mathrm{e}$ na região 2 .

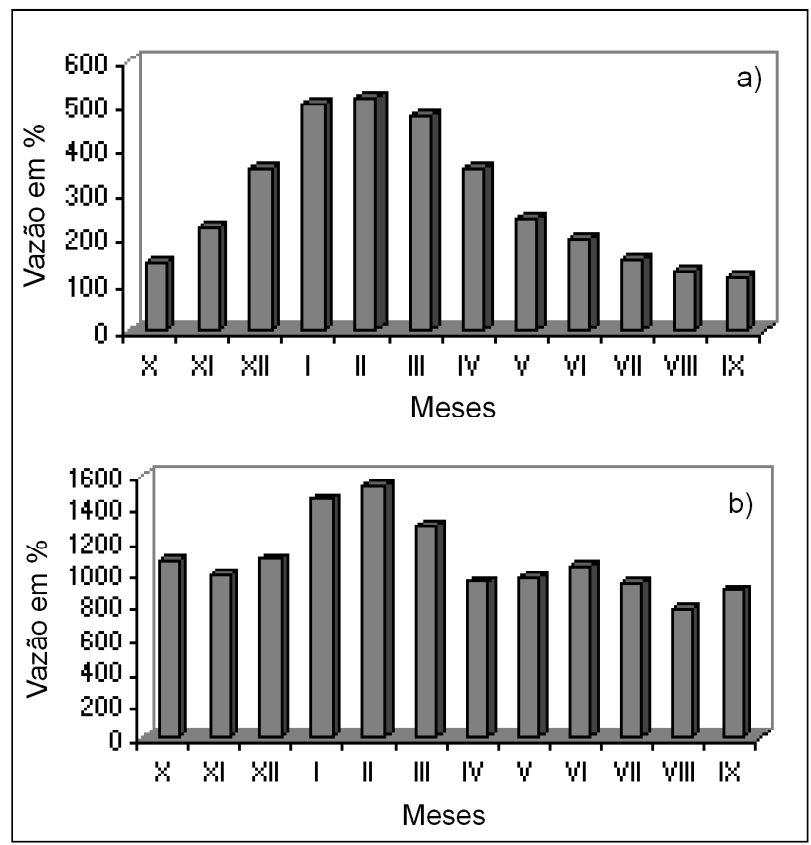

Figura 4. Distribuição de vazão mensal durante o ano nas estações Novo Ponte (a) e Capivara (b).

meira região foram obtidos os grupos janeiro a setembro e outubro a dezembro. Esta divisão não corresponde à separação clássica em hidrologia entre uma estação seca e uma chuvosa, característica do Sudeste. Em referência à Figura 4a, fica claro que ela corresponde muito mais a uma divisão em uma estação em que as vazões estão aumentando, em média, mês a mês, e uma estação em que elas estão diminuindo. Foi feita 
uma análise das correlações entre as vazões nestas duas estações do ano e a vazão anual nesta região. O coeficiente de correlação da vazão nos meses janeiro-setembro nesta região (com a vazão anual é $\mathrm{r}=0,8$, enquanto que a correlação da vazão nos meses outubro-dezembro com a vazão anual é $\mathrm{r}=0,6$.

Na Figura 5 a estão plotadas a série de médias das anomalias anuais na região 1 (linha negrito) e o fator que caracteriza as vazões dos meses janeiro-setembro nesta região (linha fina), mostrando a forte coerência entre as duas séries.

Na região 2 a análise fatorial identificou três grupos de meses: outubro a janeiro, fevereiro a abril e maio a setembro. A análise dos coeficientes de correlação mostrou que o grupo de meses maio a setembro é o mais fortemente relacionado com a vazão anual $(\mathrm{r}=0,74)$, vindo em segundo lugar o grupo de meses outubro a janeiro $(\mathrm{r}=0,53)$. $\mathrm{Na}$ Figura 5b são mostrados a variação da anomalia da vazão anual na região 2 (linha negrito) e o fator que descreve o grupo de meses maio a setembro (linha fina).

\section{Discussão}

A aplicação de análise fatorial permite gerar séries temporais anuais que resumem o comportamento das vazões nos postos fluviométricos utilizados neste estudo na margem esquerda do rio Paraná ao longo dos últimos 70 anos. A utilidade da metodologia é evidente, na medida em que ela permite identificar as tendências hidrológicas em cada região hidrologicamente homogênea. A identificação de tendências das alterações recursos hídricos é importante com as discussões sobre conseqüências de um aquecimento global da atmosfera (Showstack, 2000; Loaiciga et al., 1996; Tucci e Damiani, 1994). As séries temporais de fatores resultantes também servem como um instrumento útil de análise de teleconexões.

Da mesma forma, o agrupamento das vazões em grupos de meses ou estações "homogêneas" e o estudo da correlação entre os totais de vazão nestes grupos de meses e a vazão total no ano hidrológico permitem identificar, por exemplo, em que época do ano o impacto de uma mudança climática sobre a disponibilidade hídrica é mais importante.

\section{O EFEITO DE EL NIÑO E LA NIÑA NA VARIABILIDADE DAS VAZÕES}

Foi estudado o efeito da influência de El Niño e La Niña sobre as vazões nas regiões hidrologicamente homogêneas obtidas na seção 3. No estudo foram utilizadas as datas do El Niño e de La Niña definidos com uso do índice El Niño 3.4 a partir do ano 1950 (Kim, 2001). Segundo (Kim, 2001), durante o período 1950-1999 aconteceram 14 casos de El Niño, sendo dois fortes, seis moderados e seis fracos.

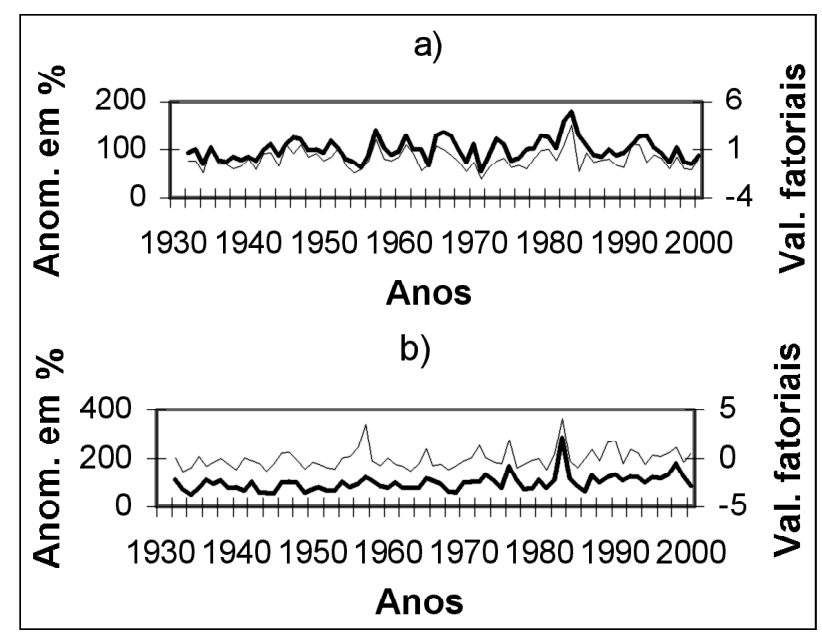

Figura 5. Variações médias anuais de anomalias de recursos hídricos (negrito) e fatores mais influentes na região 1 (a) e região 2 (b).

No mesmo período foram obtidos 12 casos de La Niña, sendo sete fracos, quatro moderados e um forte.

Uma das características mais importantes do ElNiño e da La Niña é a sua relação com seqüências de anomalias de vazão positivas ou negativas em diferentes regiões da América do Sul. Para a classificação das anomalias de médias regionais da vazão mensal foram usados os mesmos quantis que foram aplicadas para a definição de datas e intensidades os fenômenos de El Niño e La Niña (Kim, 2001). Para as vazões mensais, foram definidos como vazões anomalamente altas ou baixas quando houver um dos seguintes casos:

i. 4 meses caindo fora do intervalo 4 , sistematicamente acima (5,6 ou 7) ou abaixo (1,2, ou 3), sendo que pelo menos 3 destes moderados ou extremos;

ii. 5 ou mais meses caindo fora do intervalo 4 , eventualmente intercalados com meses isolados na média (4) (isto é: não mais que um mês " 4 " entre meses acima ou abaixo deste valor; a ocorrência de 2 meses "4" seguidos assinala o fim de um caso).

A intensidade de um caso também é classificada quantitativamente em fraca $(3,5)$, moderada $(4,6)$ ou extrema $(1,7)$. Na situação (i) (apenas 4 meses fora da média), o caso é sempre classificado ou acima do normal e fraco (5), ou abaixo do normal e fraco (3).

Na situação (ii), a intensidade de um caso quando houver 5 meses ou mais será:

- Intervalo 7 é o caso de anomalia positiva forte

- Intervalo 6 (alguns meses pode ter o intervalo 7) é o caso de anomalia positiva moderado

- Intervalo 5 (alguns meses pode ter o intervalo 6 ou 7) é o caso de anomalia positiva fraco. 
- Intervalo 3 (alguns meses pode ter o intervalo 2 ou 1) é o caso de anomalia negativa fraca.

- Intervalo 2 (alguns meses pode ficar com intervalo 1) é o caso de anomalia negativa moderada.

- Intervalo 1 é o caso de anomalia negativa forte.

Os resultados obtidos estão na Tabela 2. A segunda coluna contém a classificação dos casos de ElNiño/La Niña obtido por (Kim, 2001); a coluna 15 contém a classificação dos casos de vazões altas/baixas para a região 1 e a coluna 28 a classificação de vazões altas/baixas para a região 1, entre 1950 e 2000.

Por exemplo, na região 1 em 1952 existe uma seqüência de anomalias positivas que se inicia em março. Em maio e agosto as anomalias de vazão são normais, e como ocorrem isoladas elas não interrompem a seqüência, que se estende de março até setembro. Por outro lado, a ocorrência de dois meses normais seguidos em novembro e dezembro

Tabela 2. Classificação de El Niño/La Niña (NO/NA), das anomalias de vazão mensal por intervalos nas regiões 1 e 2 e identificação de seqüências de meses úmidos (em cinza escuro) e secos (em cinza claro).

\begin{tabular}{|c|c|c|c|c|c|c|c|c|c|c|c|c|c|c|c|c|c|c|c|c|c|c|c|c|c|c|c|}
\hline \multirow[b]{2}{*}{ Ano } & \multirow[t]{2}{*}{$\mathrm{NO} / \mathrm{NA}$} & \multicolumn{13}{|c|}{ Região 1} & \multicolumn{13}{|c|}{ Região 2} \\
\hline & & 1 & 2 & 3 & 4 & 5 & 6 & 7 & 8 & 9 & 10 & 11 & 12 & Caso & 1 & 2 & 3 & 4 & 5 & 6 & 7 & 8 & 9 & 10 & 11 & 12 & Caso \\
\hline 1950 & & & & & & & & & & & & & & & & & & & & & & & & & & & \\
\hline 1951 & 5 & 5 & 5 & 5 & 5 & 5 & 4 & 4 & 5 & 4 & 4 & 2 & 2 & 5 & 4 & 5 & 6 & 4 & 3 & 2 & 3 & 3 & 2 & 3 & 4 & 4 & 3 \\
\hline 1952 & - & 3 & 4 & 7 & 5 & 4 & 5 & 5 & 4 & 5 & 4 & 4 & 4 & 5 & 2 & 2 & 3 & 2 & 2 & 4 & 3 & 3 & 4 & 5 & 5 & 3 & 3 \\
\hline 1953 & - & 2 & 2 & 4 & 4 & 3 & 3 & 3 & 3 & 4 & 6 & 4 & 4 & - & 3 & 3 & 2 & 3 & 2 & 2 & 2 & 2 & 4 & 4 & 4 & 3 & 2 \\
\hline 1954 & 3 & 2 & 4 & 2 & 2 & 3 & 2 & 2 & 2 & 2 & 2 & 3 & 2 & 2 & 4 & 4 & 4 & 3 & 6 & 6 & 4 & 4 & 3 & 4 & 3 & 2 & 3 \\
\hline 1955 & $\mathrm{c}$ & 3 & 2 & 2 & 3 & 2 & 2 & 2 & 2 & 1 & 3 & 3 & 5 & c & 2 & 1 & 2 & 3 & 4 & 6 & 6 & 4 & 4 & 2 & 3 & 2 & $\mathrm{c}, 2$ \\
\hline 1956 & c & 3 & 2 & 4 & 2 & 4 & 5 & 4 & 5 & 4 & 3 & 5 & 6 & 6 & 2 & 2 & 2 & 5 & 6 & 6 & 4 & 6 & 5 & 3 & 2 & 2 & $\mathrm{c}, 3$ \\
\hline 1957 & c & 6 & 6 & 6 & 6 & 7 & 6 & 6 & 6 & 6 & 4 & 4 & 5 & c & 3 & 4 & 3 & 4 & 3 & 4 & 6 & 7 & 7 & 6 & 6 & 4 & $\mathrm{c}, 6$ \\
\hline 1958 & c & 4 & 4 & 3 & 4 & 4 & 4 & 5 & 5 & 6 & 6 & 3 & 2 & - & 3 & 3 & 4 & 4 & 5 & 5 & 4 & 4 & 5 & 4 & 5 & 4 & - \\
\hline 1959 & - & 5 & 3 & 4 & 4 & 3 & 4 & 3 & 4 & 3 & 3 & 4 & 3 & 3 & 4 & 4 & 3 & 4 & 4 & 4 & 3 & 4 & 3 & 2 & 2 & 2 & 3 \\
\hline 1960 & - & 4 & 4 & 5 & 4 & 4 & 4 & 4 & 4 & 3 & 3 & 5 & 5 & 6 & 4 & 3 & 4 & 4 & 4 & 4 & 4 & 5 & 4 & 4 & 5 & 5 & $\mathrm{c}$ \\
\hline 1961 & - & 6 & 6 & 6 & 5 & 6 & 6 & 6 & 6 & 5 & 3 & 3 & 2 & c & 4 & 3 & 6 & 6 & 5 & 4 & 3 & 3 & 4 & 3 & 5 & 4 & - \\
\hline 1962 & - & 5 & 5 & 5 & 4 & 4 & 4 & 4 & 4 & 5 & 5 & 5 & 7 & 5 & 2 & 4 & 6 & 4 & 3 & 2 & 2 & 3 & 4 & 5 & 4 & 3 & - \\
\hline 1963 & 6 & 5 & 4 & 3 & 2 & 3 & 3 & 3 & 3 & 3 & 2 & 2 & 1 & 2 & 5 & 5 & 4 & 4 & 2 & 2 & 2 & 2 & 1 & 4 & 6 & 4 & 2 \\
\hline 1964 & 3 & 3 & 4 & 2 & 2 & 3 & 2 & 2 & 2 & 2 & 5 & 4 & 4 & $\mathrm{c}$ & 2 & 4 & 2 & 3 & 4 & 3 & 4 & 4 & 4 & 3 & 3 & 4 & - \\
\hline 1965 & 6 & 5 & 6 & 6 & 5 & 6 & 5 & 6 & 6 & 5 & 6 & 6 & 5 & 6 & 5 & 5 & 5 & 4 & 6 & 4 & 6 & 5 & 4 & 6 & 5 & 6 & 6 \\
\hline 1966 & c & 6 & 6 & 6 & 6 & 6 & 6 & 5 & 6 & 6 & 6 & 6 & 6 & c & 5 & 6 & 5 & 4 & 4 & 3 & 4 & 4 & 4 & 4 & 5 & 5 & c \\
\hline 1967 & 3 & 5 & 6 & 5 & 5 & 5 & 6 & 5 & 5 & 5 & 4 & 6 & 5 & c & 5 & 4 & 6 & 4 & 3 & 4 & 4 & 3 & 4 & 3 & 4 & 4 & - \\
\hline 1968 & $\mathrm{c}, 6$ & 4 & 4 & 4 & 3 & 3 & 4 & 4 & 4 & 5 & 5 & 4 & 5 & - & 4 & 3 & 2 & 2 & 2 & 2 & 2 & 2 & 2 & 2 & 2 & 2 & 2 \\
\hline 1969 & $\mathrm{c}$ & 2 & 3 & 2 & 2 & 2 & 2 & 2 & 3 & 2 & 4 & 6 & 5 & 2 & 3 & 2 & 2 & 5 & 3 & 4 & 4 & 2 & 2 & 4 & 6 & 4 & c \\
\hline 1970 & 2 & 5 & 4 & 4 & 4 & 4 & 4 & 4 & 4 & 6 & 5 & 4 & 2 & 1 & 4 & 4 & 4 & 4 & 4 & 4 & 5 & 3 & 5 & 4 & 3 & 4 & - \\
\hline 1971 & c & 1 & 1 & 1 & 1 & 1 & 1 & 1 & 1 & 2 & 4 & 5 & 6 & c & 6 & 3 & 4 & 4 & 5 & 6 & 5 & 4 & 4 & 4 & 2 & 3 & - \\
\hline 1972 & 6 & 3 & 4 & 4 & 4 & 3 & 3 & 4 & 3 & 3 & 6 & 7 & 5 & - & 4 & 6 & 5 & 5 & 3 & 3 & 5 & 6 & 6 & 7 & 6 & 5 & 6 \\
\hline 1973 & $\mathrm{c}, 2$ & 4 & 4 & 4 & 6 & 5 & 4 & 5 & 4 & 4 & 6 & 6 & 4 & - & 5 & 5 & 4 & 5 & 5 & 5 & 5 & 6 & 6 & 5 & 5 & 4 & c \\
\hline 1974 & c & 4 & 2 & 5 & 6 & 5 & 5 & 5 & 5 & 4 & 5 & 2 & 3 & 5,2 & 6 & 4 & 6 & 5 & 4 & 5 & 4 & 4 & 4 & 3 & 4 & 4 & c \\
\hline 1975 & 2 & 3 & 3 & 2 & 3 & 2 & 2 & 3 & 2 & 2 & 3 & 5 & 3 & $\mathrm{c}, 3$ & 4 & 4 & 4 & 3 & 2 & 2 & 4 & 4 & 4 & 6 & 5 & 7 & 6 \\
\hline 1976 & $\mathrm{c}, 5$ & 2 & 2 & 3 & 3 & 3 & 3 & 3 & 4 & 6 & 5 & 6 & 6 & $\mathrm{c}, 5$ & 5 & 5 & 5 & 5 & 5 & 6 & 6 & 7 & 6 & 6 & 6 & 6 & c \\
\hline 1977 & $\mathrm{c}, 5$ & 5 & 4 & 2 & 4 & 3 & 4 & 3 & 3 & 4 & 4 & 5 & 4 & c & 6 & 5 & 4 & 6 & 4 & 4 & 3 & 3 & 4 & 4 & 4 & 5 & c \\
\hline 1978 & 5 & 5 & 4 & 4 & 4 & 4 & 5 & 4 & 4 & 4 & 5 & 5 & 6 & 6 & 2 & 2 & 2 & 1 & 2 & 3 & 4 & 4 & 4 & 2 & 4 & 4 & 2 \\
\hline 1979 & - & 5 & 6 & 4 & 5 & 5 & 5 & 5 & 6 & 6 & 5 & 5 & 4 & c & 2 & 2 & 1 & 2 & 6 & 2 & 3 & 4 & 5 & 5 & 6 & 5 & - \\
\hline 1980 & - & 6 & 6 & 4 & 6 & 5 & 6 & 6 & 6 & 6 & 4 & 5 & 5 & c & 4 & 4 & 5 & 5 & 4 & 4 & 4 & 5 & 5 & 4 & 3 & 6 & c \\
\hline 1981 & - & 6 & 3 & 3 & 4 & 4 & 4 & 4 & 4 & 4 & 6 & 6 & 6 & $\mathrm{c}, 6$ & 6 & 4 & 2 & 3 & 3 & 2 & 2 & 2 & 2 & 4 & 5 & 6 & 2 \\
\hline 1982 & 7 & 7 & 5 & 6 & 6 & 6 & 7 & 7 & 7 & 6 & 6 & 4 & 4 & c & 4 & 4 & 4 & 3 & 3 & 6 & 7 & 5 & 4 & 6 & 7 & 7 & 7 \\
\hline 1983 & $\mathrm{c}, 3$ & 7 & 7 & 6 & 7 & 7 & 7 & 7 & 7 & 7 & 7 & 7 & 7 & 7 & 6 & 6 & 7 & 7 & 7 & 7 & 7 & 6 & 7 & 6 & 6 & 6 & c \\
\hline 1984 & $\mathrm{c}$ & 4 & 3 & 3 & 4 & 4 & 4 & 4 & 4 & 6 & 4 & 3 & 4 & - & 4 & 2 & 3 & 4 & 5 & 5 & 4 & 6 & 5 & 3 & 4 & 5 & - \\
\hline 1985 & 3 & 6 & 5 & 5 & 5 & 5 & 4 & 5 & 5 & 5 & 4 & 4 & 3 & 5 & 3 & 4 & 4 & 6 & 4 & 3 & 3 & 2 & 3 & 1 & 2 & 1 & 2 \\
\hline 1986 & - & 4 & 4 & 3 & 3 & 3 & 3 & 4 & 5 & 4 & 2 & 2 & 4 & - & 2 & 3 & 4 & 3 & 5 & 3 & 2 & 5 & 4 & 3 & 3 & 6 & c \\
\hline 1987 & - & 4 & 3 & 3 & 4 & 4 & 4 & 4 & 3 & 4 & 4 & 4 & 5 & - & 5 & 6 & 3 & 4 & 7 & 7 & 6 & 5 & 4 & 4 & 4 & 4 & 5 \\
\hline 1988 & 1 & 3 & 4 & 4 & 4 & 4 & 4 & 4 & 4 & 4 & 5 & 4 & 4 & - & 3 & 4 & 4 & 4 & 6 & 6 & 4 & 3 & 3 & 4 & 3 & 3 & - \\
\hline 1989 & c & 3 & 4 & 4 & 3 & 2 & 3 & 3 & 4 & 4 & 3 & 4 & 6 & - & 6 & 6 & 5 & 5 & 5 & 4 & 5 & 6 & 6 & 4 & 4 & 4 & 5 \\
\hline 1990 & - & 4 & 3 & 3 & 3 & 4 & 3 & 4 & 4 & 5 & 4 & 2 & 2 & - & 7 & 4 & 4 & 5 & 5 & 5 & 6 & 6 & 6 & 5 & 6 & 4 & 5 \\
\hline 1991 & 6 & 4 & 4 & 6 & 7 & 6 & 6 & 6 & 5 & 5 & 5 & 4 & 4 & 6 & 3 & 4 & 5 & 6 & 5 & 5 & 5 & 4 & 3 & 5 & 4 & 5 & 5 \\
\hline 1992 & c & 5 & 6 & 4 & 5 & 6 & 6 & 6 & 6 & 7 & 7 & 6 & 6 & 6 & 2 & 2 & 4 & 6 & 6 & 6 & 5 & 5 & 5 & 5 & 6 & 4 & 5 \\
\hline 1993 & 5 & 4 & 5 & 4 & 5 & 5 & 5 & 5 & 6 & 5 & 5 & 2 & 4 & c & 4 & 6 & 5 & 5 & 6 & 5 & 4 & 4 & 6 & 6 & 3 & 4 & 5 \\
\hline 1994 & 5 & 6 & 3 & 5 & 4 & 5 & 5 & 5 & 5 & 4 & 4 & 4 & 4 & 5 & 4 & 4 & 4 & 4 & 4 & 5 & 5 & 4 & 2 & 3 & 4 & 4 & - \\
\hline 1995 & $\mathrm{c}, 3$ & 2 & 5 & 3 & 4 & 5 & 4 & 4 & 4 & 4 & 4 & 3 & 3 & 2 & 7 & 7 & 5 & 6 & 4 & 4 & 6 & 3 & 4 & 5 & 4 & 3 & 5 \\
\hline 1996 & c & 3 & 2 & 3 & 2 & 2 & 2 & 2 & 2 & 4 & 2 & 5 & 4 & c & 5 & 6 & 7 & 6 & 4 & 4 & 4 & 4 & 5 & 6 & 5 & 5 & 5,5 \\
\hline 1997 & 7 & 6 & 3 & 4 & 5 & 5 & 6 & 4 & 4 & 4 & 3 & 3 & 4 & 2 & 6 & 7 & 4 & 3 & 4 & 6 & 5 & 6 & 5 & 6 & 7 & 6 & $\mathrm{c}, 6$ \\
\hline 1998 & $\mathrm{c}, 3$ & 3 & 4 & 3 & 2 & 3 & 2 & 2 & 3 & 2 & 3 & 3 & 3 & c & 5 & 6 & 7 & 7 & 6 & 4 & 5 & 6 & 6 & 6 & 4 & 5 & $\mathrm{c}$ \\
\hline 1999 & c & 3 & 2 & 4 & 2 & 2 & 2 & 2 & 2 & 2 & 1 & 2 & 2 & c & 6 & 6 & 5 & 5 & 4 & 5 & 6 & 3 & 4 & 4 & 2 & 3 & c \\
\hline 2000 & - & 4 & 5 & 5 & 4 & 3 & 3 & 3 & 3 & 6 & 2 & 4 & 4 & - & 4 & 4 & 4 & 2 & 3 & 3 & 4 & 4 & 6 & 5 & 4 & 4 & - \\
\hline
\end{tabular}


de 1982 interrompe o caso iniciado em outubro de 1981; um novo caso de anomalias positivas de vazão inicia-se em janeiro de 1983.

Em cada ano, podem ocorrer um ou mais casos: em 1969 ocorreu uma seqüência de vazões baixas moderada (2) na região 1; em 1974 ocorreu uma seqüência de vazões altas fraca (5) na região 1; no mesmo ano iniciou-se uma seqüência de vazões baixas moderada (2), a qual continuou em 1975; neste ano iniciou-se uma nova seqüência de vazões baixas fraca: isto é indicado pela notação (c,3). Em 1976, a seqüência de vazões baixas continuou, e em setembro iniciou-se uma seqüência de vazões altas fraca: isto é indicado pela notação (c,5). A mesma notação é utilizada na coluna 2 para oscasosEl Niño/La Niña, cujo detalhamento mensal não é mostrado.

Na sub-região 1 no período 1950-2000 aconteceram 15 casos com vazões abaixo do normal; entre eles 1 caso é forte, 7 são casos moderados e 7 são fracos. No mesmo período na região 1 foram obtidos 9 casos com vazão abaixo do normal. Entre eles 1 caso é forte, 6 casos são moderados e 2 casos são fracos. Na sub-região 2 neste período foram determinados 16 casos com vazões acima do normal: 5 casos moderados, 1 caso forte e 10 casos fracos. Também foram determinados 13 casos com vazões abaixo do normal: 7 casos moderados e 6 casos fracos.

As relações entre ocorrências de El Niño/La Niña e casos de vazões acima/abaixo do normal nas regiões 1 e 2 estão listadas na Tabela 3. Como se pode constatar nesta tabela, os comportamentos das vazões nas duas sub-regiões não são iguais. Por exemplo, durante o El Niño dos anos 1997-1998 na sub-região 1 aconteceram vazões abaixo do normal; no mesmo período na sub-região 2 houve excesso de água. Na Tabela 3 estão mostrados os números dos casos coincidentes de vazões acima e abaixo do normal nas sub-regiões 1 e 2 com as ocorrências de El Niño e La Niña, respectivamente. Observa-se nesta tabela $9(64 \%)$ e $10(71 \%)$ das ocorrências de ElNiño coincidentes de vazões acima da normal, respectivamente, nas sub-regiões 1 e 2 . Destes, 8 (57\%) e $6(43 \%)$ têm mesma intensidade. Ao mesmo tempo, somente $60 \%$ (em ambas as sub-regiões) dos casos de vazão acima da normal são coincidentes com El Niño.

Pode-se observar na Tabela 3 que somente 7 (54\%) e $4(33 \%)$ dos casos de vazões acima da normal são coincidentes com La Niña, nas sub-regiões 1 e 2, respectivamente.

Estes resultados são coerentes com os encontrados por Kim (1998), onde a análise das relações do fenômeno El Niño e das chuvas no Rio Grande do Sul e Santa Catarina mostraram, também, cerca de $70 \%$ de coincidência entre o fenômeno El Niño e o excesso de precipitação. Em geral, as relações entre as vazões na margem esquerda de Bacia do rio Paraná e El Niño são relativamente fortes, enquanto que as relações com La Niña são praticamente insignificantes.
Tabela 3. Relações entre ocorrências de El Niño/La Niña e seqüências de vazões altas e baixas.

\begin{tabular}{|c|c|c|}
\hline Estatística & Região 1 & Região 2 \\
\hline Freqüência de El Niño & \multicolumn{2}{|c|}{14} \\
\hline Freq. de casos acima do normal & 15 & 16 \\
\hline Casos coincidentes com El Niño & $9(64 \%)$ & $10(71 \%)$ \\
\hline Casos com mesma intensidade & $8(57 \%)$ & $6 \quad(43 \%)$ \\
\hline Freqüência de La Niña & \multicolumn{2}{|c|}{12} \\
\hline Freq. de casos abaixo do normal & 9 & 13 \\
\hline Casos coincidentes com La Niña & $7(54 \%)$ & $4(33 \%)$ \\
\hline Casos com mesma intensidade & $2(16 \%)$ & $3(25 \%)$ \\
\hline
\end{tabular}

\section{PREVISÃO CLIMÁTICA DE VAZÃO}

Como foi mostrado acima, o fenômeno EL Niño acontece em média durante pouco menos que $1 / 3$ dos anos do período estudado, 1950-2000, e somente em aproximadamente 2/3 dos casos de EL Niño existe uma associação clara com anomalias de vazão fora da faixa normal nas bacias da margem esquerda do rio Paraná.

Sendo assim, embora ocorrências de El Niño forte estejam claramente associadas com vazões altas no Sudeste/ Sul sendo neste caso sinais úteis para a previsão de vazões alguns meses à frente, existem muitos casos em que o uso dos fenômenos ElNiño/La Niña é insuficiente como variável preditora da vazão.

No presente trabalho foi feito um esforço para desenvolver modelos de previsão climática mensal de vazões para os postos fluviométricos descritos na seção 2 , assim como para os postos fluviométricos adicionais de Rosana, Porto Primavera e Itaipu. Como preditores foram usados os dados mensais de TSM e $\mathrm{T}_{\text {ar }}$ também descritos naquela seção, normalizados com a Equação (11). As variáveis preditantes que foram usadas são as anomalias relativas em percentagem dadas pela Equação (7) nos postos fluviométricos de Rosana, Porto Primavera, Itaipu e as anomalias médias regionais dadas pela Equação (8) para as regiões 1 e 2 .

Conforme descrito na seção 2 , o primeiro modelo de previsão foi desenvolvido com o uso do método de analogias ou grupos análogos que no nosso caso está baseado na identificação de 20 análogos no histórico a partir dos quais se estima as médias de tendências das anomalias $\delta_{\eta_{i, t}^{+1}}$ e $\delta_{\eta_{i, t}^{+2}}$.

O segundo modelo de previsão climática de vazões avaliado está baseado na análise de correlações canônicas. Foi feita uma análise de correlações canônicas entre as anomalias mensais das vazões nos postos fluviométricos de Rosana, Porto Primavera, Itaipu, as médias das anomalias mensais de vazão da sub-região 1 e na sub-região 2 (obtidas na seção 3) e os dados de temperatura no Hemisfério Sul. 
RBRH - Revista Brasileira de Recursos Hidricos Volume 8 n.2 Abr/Jun 2003, 173-183

Tabela 4. Resultados da avaliação das previsões obtidas dos modelos desenvolvidos e as previsões de persistência e climatologia 1 mês à frente.

\begin{tabular}{lcccc}
\hline Estação & CC & A & P & C \\
& Calibração: 1941-1995 & & \\
\hline Rosana & 88 & 82 & 70 & 81 \\
Porto Primavera & 85 & 86 & 56 & 72 \\
Itaipu & 88 & 85 & 64 & 75 \\
Região 1 & 84 & 82 & 54 & 73 \\
Região 2 & 88 & 83 & 67 & 79 \\
Média & 87 & 84 & 62 & 76 \\
& Validação: $1996-2000$ & & \\
\hline Rosana & 45 & 80 & 76 & 77 \\
Porto Primavera & 50 & 80 & 62 & 73 \\
Itaipu & 55 & 80 & 65 & 77 \\
Região 1 & 52 & 82 & 80 & 68 \\
Região 2 & 43 & 70 & 63 & 82 \\
Média & 49 & 79 & 59 & 75 \\
\hline
\end{tabular}

Uma vez que o objetivo era a obtenção de um modelo de previsão, os dados de anomalias de vazão foram defasados de 1 e 2 meses dos dados de temperatura.

Foram calculadas e avaliadas as previsões da vazão média mensal 1 e 2 meses à frente obtidas por meio de ambos os modelos para um período de calibração entre 1940 e 1995. Para o período de 1996 e 2000 os dois modelos foram avaliados. Os resultados de avaliação do método de analogias (A) e do método de correlação canônica (CC) foram comparadas com os que seriam obtidos caso fossem utilizadas também como "previsões" as anomalias de vazão baseadas em persistência (P) (isto é: prevê-se para 1 ou 2 meses a frente um valor de $\eta$ igual ao observado no presente) e climatologia (C) (ou seja: prevê-se sempre $\eta=100 \%$ ). Estas avaliações estão mostradas nas Tabelas 4 e 5 . Conforme mencionado acima na seção 2 , uma previsão é considerada acertada quando cai em uma faixa de +1 desvio-padrão do valor correto. O resultado das avaliações é dado em porcentagem de previsões acertadas.

Verifica-se na Tabela 4 que, no período de validação, as previsões do modelo de correlações canônicas são um pouco superiores às do modelo de analogias (87\% contra $84 \%$ ) e superiores ao uso tanto da persistência quanto da climatologia. Os resultados são dramaticamente diferentes no período de validação, quando o desempenho do método das correlações canônicas se torna pior que o uso de persistência ou da climatologia, e no qual o método de analogias é claramente vencedor.

$\mathrm{Na}$ Tabela 5 são mostrados os resultados das avaliações das previsões para 2 meses à frente. Novamente, durante o período de calibração o método de correlações canônicas revela-se superior, e mais uma vez este resultado reverte-se no período de validação. Neste caso, a previsão
Tabela 5. Resultados da avaliação das previsões obtidas dos modelos desenvolvidos e as previsões de persistência e climatologia com antecedência de 2 meses.

\begin{tabular}{lcccc}
\hline Estação & CC & A & P & C \\
& Calibração: & 1941-1995 & & \\
\hline Rosana & 86 & 77 & 67 & 81 \\
Porto Primavera & 86 & 78 & 56 & 72 \\
Itaipu & 89 & 76 & 63 & 75 \\
Região 1 & 86 & 77 & 54 & 73 \\
Região 2 & 89 & 75 & 67 & 79 \\
Média & 87 & 77 & 61 & 76 \\
& & & & \\
\hline Rosana & 62 & 62 & 68 & 77 \\
Porto Primavera & 47 & 80 & 58 & 73 \\
Itaipu & 55 & 58 & 58 & 77 \\
Região 1 & 47 & 73 & 80 & 68 \\
Região 2 & 53 & 57 & 56 & 82 \\
Média & 53 & 68 & 64 & 75 \\
\hline
\end{tabular}

baseada na climatologia resulta vencedora durante o período de validação.

As previsões das anomalias de vazão mensais podem ser transformadas em previsões de vazão mensal por meio da Equação (7), para todas as estações fluviométricas de cada região analisada. A Figura 6 mostra exemplos do desempenho do método de analogias para a previsão de vazões 1 mês à frente em 3 estações fluviométricas: Itaipu (a), Corumbá (b) e Salto Osório (c). O desempenho obtido e mostra a possibilidade aplicar o método de analogia por exemplo, no setor elétrico.

\section{CONCLUSÕES}

O estudo mostrou a possibilidade do uso de análise fatorial para a regionalização hidrológica e identificação de meses estatisticamente homogêneos. Os períodos de meses estatisticamente homogêneos encontrados para as sub-regiões 1 e 2 são diferentes das tradicionais estações de "seca" e "cheias" muito comuns em estudos hidrológicos. A análise dos grupos dos meses com variações semelhantes de vazões nas duas sub-regiões mostrou que o período janeiro a setembro são os mais importantes na composição da variação da vazão anual para a sub-região 1 e o período maio a setembro na composição da variação da vazão anual para a sub-região 2 .

As sub-regiões obtidas têm boa correspondência com os processos atmosféricos, a primeira sub-região estando localizada acima de $22^{\circ} \mathrm{S}$ (zona tropical) e a segunda abaixo de $22^{\circ} \mathrm{S}$ (zona subtropical). A análise mostrou a diferença entre as distribuições das normais climáticas de vazões mensais dos rios nas sub-regiões encontradas. Na primeira sub-região a vazão 


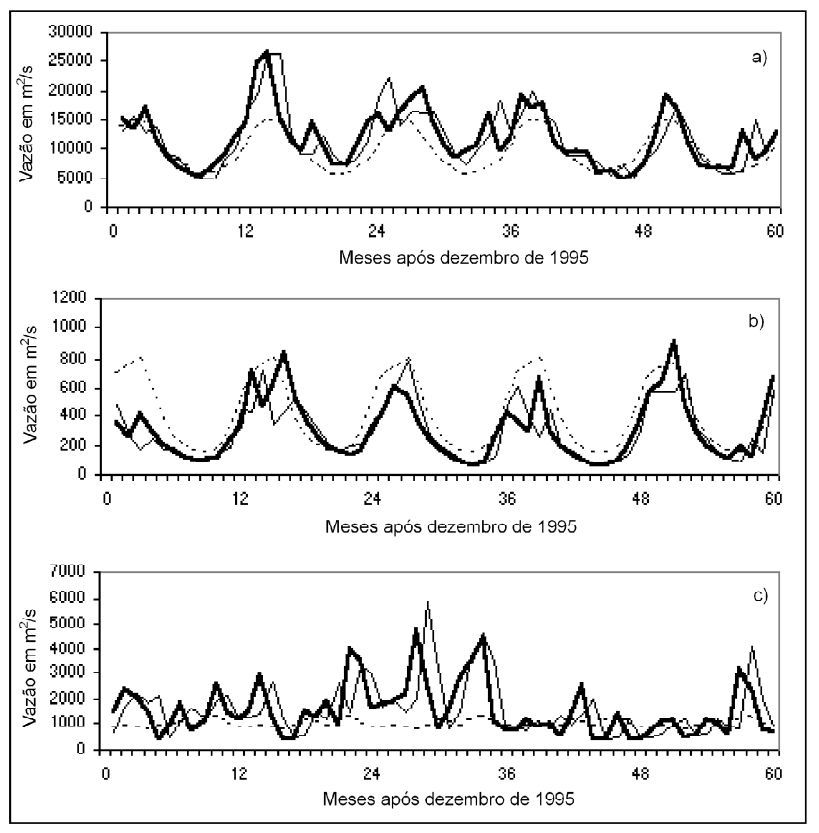

Figura 6. Séries de vazões mensais observadas (linha espessa), previstas (linha fina) e normas climáticas (linha pontilhada) nas estações fluviométricas de Itaipú (a), Corumbá (b) e Salto Osório (c).

mensal máxima é no mês de fevereiro e a mínima em setembro; na segunda sub-região existem três picos, sendo o principal em fevereiro e os secundários em outubro e junho. Há também três mínimos: em abril, agosto e novembro. A análise das variações de vazões nas sub-regiões encontradas mostrou que na primeira sub-região até o ano de 1980 não existe uma tendência linear de crescimento ou diminuição das médias anuais, mas nos últimos 15 a 20 anos as vazões nesta sub-região estão diminuindo. Na segunda sub-região, há uma tendência de crescimento nas vazões médias anuais.

O estudo do efeito da influência de El Niño sobre as vazões nas sub-regiões hidrologicamente homogêneas mostrou que $64 \%$ e $71 \%$ dos casos de EL Niño foram associados com vazões acima do normal nas sub-regiões 1 e 2 , respectivamente. O efeito da influência de La Niña é praticamente insignificante: só $55 \%$ e $33 \%$ dos casos de ocorrência deste fenômeno foram associadas a vazões abaixo do normal.

Foram desenvolvidos métodos de previsão climática das vazões mensais baseadas na analogia, correlação canônica. Com o uso dos modelos desenvolvidos foram calculadas e avaliadas as previsões da vazão média mensal 1 e 2 meses à frente para um período de calibração entre 1940 e 1995 e para o período de validação entre 1996 e 2000. Os resultados de avaliação dos modelos foram comparados com as previsões de persistência e climatologia. A validação dos métodos de previsão climática da vazão mensal mostrou um bom desempenho de método de analogia diante de métodos baseadas na correlação canônica, persistência e climatologia com antecedência um mês. Porem, as previsões obtidas através de modelos desenvolvidos para dois meses na frente já não tem vantagem diante de climatologia.

\section{REFERÊNCIAS}

BARNET, T. P. \& PZEISENDORFER, R. (1987). Origin and levels of monthly and seasonal forecast skill for United States Surface Air Temperature determined by canonical correlation analysis. Monthly Weather Review, vol. 115, p. 1825-1850.

GENTE, J. L.; IRIBARREN, G. P. \& MECHOSO, C. (1998). Recent increasing tend in streamflow of rivers in Southwestern South America. Journal of Climate, vol. 11, p. 2858-2862.

GNANADESIKAN, R. (1997). Methods for statistical data analysis of multivariate observations. $2^{\circ}$ ed. New York; John Wiley.

GUIETTO, M. A. \& BERRY, G. J. (1996). Anomalías Estacionales de los Volúmenes de la Cuenca del Plata en Puerto Bermejo, Posadas y Corrientes y su Relacione con El Niño. In: Anais do VII Congresso Argentino de Meteorologia. Buenos Aires, p. 139-140.

KENDAL, M. G. (1980). Multivariate analysis. $2^{\circ}$ ed. London: Charles Griffin \& Company.

KIM, I. S. (1998). Análise da variabilidade e variação dos índices El Niño, Oscilação Sul e chuvas nos Estados do Rio Grande do Sul e Santa Catarina do Brasil. In CD-ROM, X Congresso Brasileiro de Meteorologia. Brasília.

KIM, I. S. (2001). Sobre a definição das datas de El Niño e La Niña. In: $C D-R O M$, IX Congresso Latino-Americano e Ibérico de Meteorologia. Buenos Aires.

LOAICIGA, H. A.; VALDES, J. B.; VOGEL, R.; GARVEY, J. \& SCHWARZ, H. (1996). Global warming and the hydrologic cycle. Journal of Climate, vol. 174, p. 83-127.

MECHOSO, C. R. \& IRIBARREN, G. P. (1992). Streamflow in Southeasthern South America and Southern Oscillacion. Journal of Climate, vol. 5, p. 1535-1539.

SHOWSTACK, R. (2000). Advisory Committee Releases U. S. National Assessment on Potential Consequences of Climate Change. EOS, vol. 81, n. 47, p. 561-562.

TRENBERTH, K. E. (1997). The definition of El Niño. Bull. of the Am. Met. Soc., vol. 78, n. 12, p. 2771-2777.

TUCCI, C. E. M. \& DAMIANI,. (1994). Potencial impacto da modificação climática no rio Uruguai. Revisa Brasileira de Engenharia - Caderno de Recursos Hídricos, vol. 2, p. 5-34. 
Variability and Climate Forecasting of Flows on the Left Bank of the Upper Paraná Basin

(Brazil)

\section{ABSTRACT}

A regionalization study of the left bank of the Upper Parana' River Basin (Brazil) was performed by means of factor analysis, resulting in two bydrologically homogeneous sub-basins. Factor analysis was also used to group months into seasons, in order to identify the season that contributes most to the mean annual flow. El Niño and La Niña influences on streamflow on the left bank of the Upper Paraná River Basin (Brazil) were analyzed. Moreover, analogy and canonical correlation methods using information about sea-surface and air temperature at selected points of the Southern Hemisphere (with negligible correlations to each other) were utilized to develop and validate monthly climate forecasting models for flows.

Key words: regionalization; climate forecasting. 